\title{
ON THE ORIGIN OF CIRCULAR AND HEXAGONAL FORMATIONS IN GALAXIES
}

\author{
Yu.N.Efremov \\ Sternberg Astronomical Institute, Moscow University, Moscow, 119899, Russia,
}

\begin{abstract}
The round and arc-shaped formations are known in some galaxies, the Bubble complex (the Hodge object) in NGC 6946 being the most remarkable. The rim of the complex has a form of a regular arc in which a part of a hexagonal structure is embedded. A similar morphology is recognized in NGC $\mathbf{7 4 2 1}$ galaxy, in that part of its rim which is leading in the galaxy motion through the intergalactic gas, as suggested by the bowshock appearance of the HI halo of the galaxy. A hexagonal shape is also found in NGC4676A galaxy. The HII radial velocities across the Bubble complex are compatible with its retrograde rotation and drift, which are characteristic for solitary vortices known in nonlinear hydrodynamics. The drift motion may explain the location of the Bubble complex at the tip of the largest elliptical HI hole in NGC 6946. The hexagonal vortices in the atmospheres of Jupiter and Saturn are within the gas streams what seems to be suggestive as well. It is conjectured that the hexagonal rims of stellar systems might be relicts of flat segments of the shock
\end{abstract}


wave produced by the ram pressure. The giant stellar arcs in NGC 300 and M33 are associated with the energetic X-ray sources P42 and X-4 respectively (fig. 11), and these might be the relics of Hypernovae.

Key words: galaxies: individual (NGC 6946, M83, NGC 4449, NGC 7421, NGC 4676, LMC); stellar complexes; large scale star formation; spiral arms; vortices; ram pressure; Hypernovae

\section{Introduction}

Most stellar systems concentrate to the center of mass and have more or less roundish overall shape, without sharp rims (excepts the shell galaxies and some other products of the galaxy interactions). It is natural, for their shape is mostly determined by interplay of gravitation and rotation (internal motions). However there are stellar complexes with sharp and very regular, arc-shaped rims, which might have a peculiar origin (Efremov 2001, 2002). Some galaxies are known also to have sharp circular rims or the polygonal shape. Here we give examples of such geometrically regular systems and discuss hypotheses on their origin.

\section{The peculiar stellar complex in NGC 6946}

The Bubble complex (the Hodge object) in NGC 6946 was discovered by Hodge (1967) and independently by Larsen and Richtler (1999). Its origin is a subject of discussions. The complex is really unusual (Fig. 1), especially because its western rim is sharp and has the shape of the regular arc with radius $300 \mathrm{pc}$. The oldest stars are 30 Myr old and the rate of star formation there is 1-2 order of magnitude higher than that in the Local complex (Gould Belt). Another peculiarity of the complex is the very massive

young cluster near (yet not at) the center, quite similar to ones known in the interacting galaxies (Larsen et al., 2001; Larsen et al., 2002).

The complex rim is sharp and circular only at the West, what was the motivation to suggest the origin of the complex under the impact of a cloud moved along the oblique trajectory from East to West (Efremov, 2002). The highest light absorption is 
also observed along this rim (Larsen et al., 2002). Other suggested scenarios were the extreme case of the normal star formation (Elmegreen et al., 2000) and the triggering by a Super-supernova (Hodge, 1967).

The kinematics of HII gas inside and near the complex was studied with the long slit at the $6 \mathrm{~m}$ telescope (Efremov et al., 2002). The radial velocity curves along all three slit positions demonstrate many perturbations, some of which may be interpreted as the expanding shells or semishells. The most evident feature was considered to be the semishell with the velocity of expansion about $120 \mathrm{~km} / \mathrm{s}$ and size of about $300 \mathrm{pc}$, its center is 7" East of the massive cluster. Apart from these disturbances, there is the wide positive bump encompassing all the complex, with the average velocity about $20 \mathrm{~km} / \mathrm{s}$ larger than that of the surrounding field. It is worth noting that this velocity is quite close to the velocity of the young massive cluster, which was determined independently at 10-m and 6-m telescopes and is $150 \mathrm{~km} / \mathrm{s}$ (Larsen et al., 2001; Efremov et al., 2002).

We conjectured that the peculiarities of the velocity curves which are seen in Figs. 4, 5 and especially Fig. 6 in the paper by Efremov et al. (2002) may be explained by the suggestion that the gas motions in the complex are mostly connected with vortices, whereas the positive bump might reflect the bulk drift motion of the complex in the plane of the galaxy. The velocity curve for PA $-37^{\circ}$ and the Western (undisturbed) parts of the curves for PA $83^{\circ}$ and PA $29^{\circ}$ demonstrate the linear gradients, which are different for different position angles in a way compatible with the solid body rotation in the plan of NGC 6946, to the direction opposite to the rotation of the galaxy.

The retrograde rotation and drift are expected for the Rossby soliton in the galaxy disk (Korchagin and Petiashvili, 1985; Korchagin and Ryabtsev, 1991). Considering the positive bump of HII velocity has the same value as the main cluster velocity and both deviate from the rotation of the galaxy, the prograde drift of the complex seems to be a possible interpretation. The amplitude of the wide bump being about $20 \mathrm{~km} / \mathrm{s}$ in radial velocity and and the inclination of the galaxy plane to the sky being $32^{\circ}$, this translates to the velocity in the plane of the galaxy about $40 \mathrm{~km} / \mathrm{s}$.

It is possible that the drift of the complex in relation to the stuff of the galaxy disk may explain the strange absence of the HI hole around it. Moreover, overlay of the 
Kamphuis (1993) map of HI hole in NGC 6946 and the galaxy image from the DSS (Fig. 2) demonstrates the position of the complex at the Southern tip of the supergiant elliptical $(6.5 \times 2.9 \mathrm{kpc})$ HI hole $(\# 13$, surely) outside the latter. The geometry of the situation, the direction and velocity of the suggested complex drift imply that 30 Myr ago (the age of the first burst of star formation in the complex) the complex was located well inside the hole \#13. One may suggest that formation of the hole \#13 and the complex were triggered by the same HVC impact. This hole is associated with the high velocity gas region \#12 (Kamphuis, 1993, Table 2) which has energy $8 \times 10^{53} \mathrm{erg}$ and mass $3.1 \times 10^{7} M_{\odot}$, whereas the missing mass in the hole \#13 is $22.3 \times 10^{7} M_{\odot}$ (Kamphuis, 1993, Table 1). The oval shape of the hole \#13 may reflect the way of the Bubble complex through the gaseous disk and the present day location of the complex near the hole tip (yet outside it) may indicate its recent arrival to this point and the time needed to form the expanding HI hole around.

Our hypothesis implies that under some conditions a cloud impact may trigger the gaseous vortices in the disk of the galaxy. There is no large scale perturbation of the NGC 6946 galaxy rotation curve needed in the theory to trigger the solitary vortex (Rossby soliton). We suggest a special origin of a vortex, not connected with the bulk dynamics of the galaxy. The loop of the velocity field, seen in the region of the Hodge object (Bonnarel et al. 1988) may be result of the initial local perturbation. The vortices indeed formed in the tail of the impacting cloud in the Santillan et al. (1999) models of the HVC impact to the magnetized galactic gas disk. May such gas motions trigger then the star formation? At any rate, the star formation might be confined within the long standing gas vortex.

The radial velocity curve for PA $83^{\circ}$ (Figs. 4 and 6 in Efremov et al. 2002) contains the deep dip which was explained in that paper as the expanding semishell. The wide positive bump is centered on the massive cluster, yet the dip is in the center of the additional more narrow positive bump to the East of the complex center. This configuration must be explained. One may suggest that here we have not the semishell expanding to us yet two adjacent vortices with very fast and opposite rotation, the situation expected in cosmic hydrodynamics and known as von Karman vortice street (Chernin, 1996). 
The expanding semishell might be connected with the position of the pressure source outside the plane of symmetry of the galactic plan (what is compatible with the Hypernova hypothesis for the semishell origin, Efremov et al. 2002). However, the plot of $V_{r}$ for such a structure should be a sinusoid, whereas we rather see two straight lines,

especially certain for the Eastern side of the $V_{r}$ dip. These two lines suggest existence of two vortices, rotating in opposite directions. They might be parts of "von Karman vortice street" in the tail of the impacting cloud, such as ones seen in model by Santilan et al. (1999). Such vortices are not the full rings and, apart from this, the rotation in the the galactic plane easy explains why no line splitting is seen over the dip.

\section{Ram pressure and star formation}

The best way to understanding a peculiar property is to find other examples of systems which share the property. So we have been looking for circular rims everywhere. Indeed, 130-degree long arc of the Western rim of the Bubble complex has analogies in the the Quadrant and Sextant arcs in the LMC, yet also in rims of a few galaxies.

The galaxies with the circular rims are known, indeed. It is DDO 165 in the M81 group, the Southern rim of which is sharp and arc-shaped (Efremov, 2001). In the same M81 group, the Ho II galaxy has the outer HI isolines of the characteristic comet-like shape (Bureau and Carignan, 2002), and SE part of HI halo of this galaxy is bordered by the perfect arc of a circle. These authors suggest the action of the ram pressure as the reason for this shape. Even earlier the same suggestion was proposed by Ryder (1997) to explain the similar shape of HI halo of NGC 7421 galaxy and it is surely true for NGC 2276 galaxy as well (Fig. 3).

The side of the stellar disk of NGC 7421 turned to direction of the movement is evidently enhanced and sharp-bordered. This is even more evident for NGC 2276 galaxy, where the HII isolines (Gruendl et al. 1993) and even the stellar disks are shaped in accordance with the Mach-cone like appearance (Fig. 3) of the galaxy at $1.49 \mathrm{GHz}$ (Hummel and Beck, 1995).

These are the clear evidences for the star formation triggered by the ram pressure 
and still preserved the characteristic bow-shock shape. We may therefore suggest that the southern rim of DDO 165 (fig. 4) is also relict of the star formation triggered by the ram pressure in the bow-shock shaped edge of the galaxy gas (Efremov, 2002).

The conclusion follows that the 130-degree long western arc of the Bubble complex in NGC 6946 may also be relict of the star formation in the bow shock at the interface of the impacting cloud and NGC 6946 gas disk. The same explanation may be true also for the LMC arcs in the LMC4 region, their occurrence in the same (NE) region of the galaxy being explained by observation that the NEE edge of the LMC is leading in the LMC motion and is the first to meet the clouds of the Galactic halo (Efremov, 2002).

We noted that this may be confirmed by the bow-shock appearance and orientation of the X=ray emission around SNR N63 (Chu, 1999) localized at NE of the LMC4 supershell, near the NE edge of the LMC gaseous disk (Fig. 5 and 11) - it may be shaped by the ram pressure owing to the LMC motion through the halo of the Galaxy. The line drawn through the X-ray maximum under the position angle of the LMC proper motion $79^{\circ}$ (Marel et al., 2002) is close to the axis of the symmetry of X-ray nebula (Fig. 5). The sharp NE edge of the distribution of $\mathrm{HI}$ in the LMC is in accordance with this.

\section{Hexagonal rims connected with ram pressure?}

The close examination of the Bubble complex rim under different image contrasts reveals that at least half of its rim is confined by segments of hexagon inscribable into a circle. This is seen for the "leading" (Western) part of the complex in the HST images, whereas in NOT images all the complex, after application of the non-monotonic characteristic curve, display the pentagon shape (Fig. 1).

There are also the examples of galaxies with hexagonal or pentagonal rims. One may note that the leading rim of NGC 7421 is also outlined by the segments of hexagon inscribable into the arc of the circle. Something like this is seen also in NGC 2276

image yet not so definitely (Fig 3). The similarity of the leading edge rims of these two galaxies and the western rim of the Bubble complex is evident. These galactic rims are certainly shaped (in the end) by the ram pressure and that of the Bubble complex is 
plausibly too. Anyway, a number of other galaxies (especially in the Virgo cluster) are known to have signatures of the ram pressure action yet have no the hexagonal rims. This may be explained by observation that the galaxy must be seen pole on, in order the polygonal shape of its disk would be noted, as it is the case for NGC 7421 and NGC 2276. Otherwise, the hexagonal shape may be a transient property of the ram pressure shaped gaseous cloud, which may not be necessarily preserved in the stellar distributions.

Other astronomical observations may be the clue to the issue. There is known at the Saturn North pole the hexagonal cloud feature discovered in Voyager close-encounter images (Allison et al., 1990). These authors interpreted the hexagonal cloud as a stationary Rossby wave. They note also that the wave is embedded within a sharply peaked eastward jet (of 100 meters per second) and appears to be perturbed by at least one anticyclonic oval vortex immediately to the south.

In result of browsing of available images of the Jupiter Red spot we have noted that sometimes the vortex bordered by segments of hexagon is developing inside the Spot, while the leading rim of the Red spot has acquired the shape of two straight lines, as it is seen in the image obtained by Voyager in 1979 (Fig. 6). The turbulent vortices behind the Spot are well seen too (see http://antwrp.gsfc.nasa.gov/apod/ap020205.htm]).

As the hexagonal cloud at Saturn, the Red spot is known to locate in the stream of the fast moving gas of the Jupiter atmosphere, yet it is the persistent vortex not sharing the movement of encompassing gas. The analogy with the ram pressure action to the galaxy moving through intergalactic gas is evident.

The experiments with rotating shallow water demonstrated the arising of the structure interpreted as the Rossby solitary vortex, which emerges because of the self-organization of dissipation system far from the equilibrium state (Wang et al. 2001). These authors concluded the experiment is a model of the Jupiter Red Spot. In fact, the similar experiments were carried out by many authors (see the references in Korchagin and Ryabtsev, 1991), and the suggestion that Jovian Red Spot is a stationary nonlinear soliton-like vortex was advanced long ago by Maxworthy and Redekopp (1976). It is worth noting that, as was demonstrated by Korchagin and Ryabtsev (1991) the hydrodynamical theory of solitary vortices is applicable to a stellar galactic disk as well. 
The hexagonal and asymmetrical galaxies are seen also in the Hubble Deep Fields. Some samples extracted from the ingenious site of S.Gwyn

(http://astrowww.phys.uvic.ca/grads/gwyn/pz/hdfs/spindex.html) are given in Fig.

7. These galaxies are mostly in small groups and the ram pressure origin of the hexagonal shape is plausible. It is worth noting that in some galaxies (293N and $222 \mathrm{~S})$ there are twin blue spots at the presumably rear (in motion) edge. These spots might be the result of the star formation in the vortex couple known to locate behind the fast moving body (see also Fig. 1).

It is possible that the hexagonal shape might be the transient property of the persistent vortices, which are objects of the ram pressure. It is a result of self-organization, reminding by the resulting shape (yet not by physics of origin) the cells of Benard. If the star formation occurred in the shock wave while its front was flat, the facing to the stream part of the stellar system rim would preserve the hexagonal shape.

Anyway, note the Benard-cells like appearance of the unique Honeycombe feature in the LMC, located at 100 light year from the SN1987A. The structure consists of two dozen adjacent gaseous cells, most of similar size 3 pc (Noever, 1994; Meaburn et al. 1995) and the high velocity gas motions observed along the cell walls (in direction perpendicular to the cell planes) remind the convective motions along the walls of the Benard cells. Redman et al. (1999) suggest that the cells were formed owing to the Rayleigh-Taylor instability in the shell of the old SNR, whereas Noever (1994) noted that regularities of the polygons of the Honeycomb follow some laws of the statistical crystallography.

Chernin (1996) noted that it must be explained why the largest known eddies in the Universe are galaxies, and one may wonder if the whole galaxy might be considered as the Rossby soliton. A far going idea of such a kind was published, indeed (Dubrovsky, 2000). In accordance with this suggestion, the rotational velocity of a galaxy is determined by the vortex instability rate and not the central mass, so the hidden mass may not be necessary. 


\section{$5 \quad$ What are the dark rings?}

The dark rings surrounded by arcs of clusters are known in M83 (Efremov, 2001) and NGC 4449 (Bothun, 1986), of about 500 pc diameters, like the Hodge complex (Fig. 8). They are seen in some other galaxies too (NGC 6946, NGC 2207). At the first glance, these rings might be considered the examples of the star formation triggered by the gravitational instability in the swept up gas shell, more so the distances between clusters in M83 Western ring being remarkably equal. However, a swept up expanding shell should leave the inner space of the ring empty from gas and dust, whereas there is dust; the central clusters are not seen in M83 rings (the bright object inside the Southern ring should be the foreground star), yet are in NGC 2207 and NGC 6946 (Fig.8). The visible darkness of the NGC 4449 complex inside the ring is confirmed by the photometric data (Hitchock, Hodge 1968). If so, the gas density inside such rings is higher than in surroundings and the circular shape may suggest these rings are gaseous (inspiralling) vortices with the enhanced column gas density at least along their rims, where the star formation was effective.

The multicolor observations could find the parameters of the light absorption inside these rings. It would not be surprising to find these to be unusual, like it is the case for the dark cone centered on the star cluster in the highly peculiar stellar complex in NGC 2207 (Elmegreen et al. 2000 - see Fig. 8). The very high resolution HI and CO observations could also solve the problem. To find the real absence of even old stars inside the dark rings would be tremendous yet surely improbable possibility. The features of this kind found in the cores of a few galaxies are much smaller (Lauer et al., 2002)

Considering that inside the dark rings in NGC 6946 and NGC 2207 the central objects (clusters?) are seen (and there is a guess for this in NGC 4449, Fig. 8), these rings may still be examples of the swept up shells, after some interactions of the shock waves. The smaller scallops at the inner side of both rings in M83 (the only images with good resolution) may also suggest this. Anyway, the known tested examples of the star

formation in the swept up shells, such as in IC 2574, are quite non-similar to these rings, as well to other formations discussed here (see Efremov, 2002). 


\section{Other hexagonal galaxies}

The hexagonal shape is well known for the rings surrounding some galaxies and also for bulges of some S0 galaxies. The list of such galaxies and discussion of their nature was recently given by Chernin et al. (2002). They noted that the galactic rings are intimately related to spiral structure, as Buta and Combes (1996) demonstrated, and the origin of the hexagonal structure may be the same as of the polygonal arms known in many grand design galaxies (Chernin 1999, Chernin et al. 2000).

As Chernin et al. (2002) noted, there is the major similarity between hexagon structures and polygonal shape of the spiral arms: in both structures the angle between adjacent straight lines is about $2 \pi / 3$ and the lengths of the straight segments are equal to the radius of encompassing circle or to the local radius of curvature of the logarithmic spiral. Chernin (1999) suggested that the local flattening of the spiral front on the space scale of local radius of curvature may be due to stability of a flat shock wave against any weak perturbations that disturb its front surfaces. On this basis, Chernin et al. (2002) concluded that the hexagons are also made of flat segments of shock fronts.

This suggestion may explain the hexagonal-shaped rim of the Bubble complex and of the galaxies which, like NGC 7421 and NGC 2476, are object of the ram pressure. The occurrence of the straight segments of rims of these galaxies at the shocked side only is especially indicative.

However, there are rare galaxies which are hardly objects of the ram pressure yet whose disks have hexagonal shape. The long known example is NGC 6776 (Sansom et al. 1988) which is considered to be E galaxy but is certainly S0, being rotating. Another is NGC 1637, the three-armed well isolated spiral (Fig. 9). There is no other galaxies within $1^{o}$ from it (Block et al. et al. 1988).

Another problem is the peanut shape of bulges of some S0 galaxies, the best samples being NGC 7020 (Buta, 1990) and IC 4767 (Whitmore and Bell, 1988). These galaxies look like the inclined hexagons. The proposed explanations were the interactions with other galaxies or specific shapes of stellar orbits. The key issue here is the orientation; the visible prolate hexagons may be intrinsically prolate thick bulges seen edge-on or they may be the hexagonal disks seen under angle. The former possibility seems to be 
easier explainable by the orbit orientations for the NGC 7020 case (Buta 1990). Also, Merrifield and Kuijen (1999) argued that the boxy and peanut-shaped bulges of some galaxies are galactic bars viewed from the side.

We found an example of the hexagon where the edge-on orientation is sure. It is NGC 4676 ("the Mices") interacting galaxies case. The image obtained recently by the HST with the Advanced Survey Camera (see http://oposite.stsci.edu/pubinfo/pr/2002/11/ after editing reveals the hexagonal shape of the Northern (NGC 4676A) galaxy, whereas the existing kinematical data indicate it is seen edge-on (Yun and Hibbard, 2001). This inclination follows also from the thin and straight appearance of the long tail of the NGC 4676A. The tidal tail should be intrinsically wide and planar, so in the Mices system it is seen edge-on. The overlay of CO isolines from Yun and Hibbard (2001) and the HST ACS image (Fig.10) demonstrates that not all dust clouds seen in NGC 4676A are connected with $\mathrm{CO}$ emission in the galaxy and vice versa. This might be partially explained by the localization of some dust clouds in front of the galaxy body.

These interacting galaxies are in the Coma cluster and may be objects of the ram pressure. We suggest that the curved cone-like plume at the North of NGC 4676B is just the manifestation of action of the ram pressure onto the leading edge of the galaxy. Overall structure of the system may display the orbital motion of the Mices. The blue color of the turned cone tip of NGC 4676B is surely result of the star formation, triggered by the ram pressure. If so, one may still wonder if the ram pressure was participated in shaping the NGC 4676A galaxy as well.

\section{$7 \quad$ Stellar arcs and Hypernovae}

The best known arc-shaped complexes are the LMC4 group of arcs 200 - 300 pc in radii, noted first by Hodge (1967). The concentration of arcs near to each other (Fig. 11) was explained by their origin from the objects, ejected from the massive 2 Gyr old cluster NGC 1978 in the same area. It was suggested that the progenitors of GRB/Hypernovae are binaries of the compact objects formed owing to dynamical interaction of stars and/or their remnants within the dense cluster and then ejected from it. We argued that this is 
the main channel of formation of binaries with compact components (Efremov, 2001b).

The same idea is now accepted to explain the concentration of X-ray binaries to the globular clusters (White et al. 2002). According Kundu (2002), 40 percent of the brightest LMXBs (which are suggested to be the black hole accretors) in the elliptical galaxy NGC 4472 are associated with the globular clusters. We have noted the concentration of the X-ray binaries in the region of the LMC4 (Efremov 2001b and references therein) and may now conclude that idea on the origin of progenitors of the LMC4 arcs in the NGC 1978 cluster is still alive.

The origin of the LMC system of arcs due to the ram pressure onto the surface of the impacting cloud was also considered (Efremov, 2002). The physically similar situation arises after interaction of a dense and cold enough cloud with the blast wave from the powerful external explosion. The large increase in pressure leads to the compression of the cloud, most rapid at the face, exposed to the blast wave, and the bow shock may appear along this side (McKee and Cowie, 1975). The observational data discussed above demonstrate that the triggered star formation may result, the bow shock appearance being preserved in the distribution of the young stars.

The 400 pc in size arc of the bright stars, noted in Efremov (2001), and now known as the complex AS102, in the spiral arm of the NGC 300 galaxy may be result of such an event (Fig. 11). The brightest in the galaxy X-ray source, $\mathrm{P} 42=\mathrm{H} 13$, which is classified as X-ray accreting binary system including a black hole (Read and Pietsch, 2001), is near the complex at its convex side and exactly on the axis of symmetry of the stellar arc (Efremov, 2002). Considering the age of the complex ( 5 Myr, Kim et al. 2002) and its distance to P42, the energy needed to compress the paternal cloud to AS102 stellar complex should be that of a Hypernova.

The last example is the arc of the OB-association HS137 in M33, noted in Efremov (2001). It is also known as the HII region IC133 (Fig. 11). This arc encompasses the HI hole \#31, coordinates of which are the same as of X-ray source M33 X-4 (Schulman and Bregman, 1995). This source was investigated recently by Okada et al. (2001) who found it to be the young SNR with energy considerably higher than those of SNRs in the Milky Way. This X-ray source is the only in M33 which is suggested to be physically 
associated with a HI hole (Schulman and Bregman, 1995).

The arc-like appearance of the AS 102 complex formed by the outer pressure to the paternal cloud is natural. However, for IC133 complex we evidently deal with the classic model of the star formation in the swept up shell, and the stellar arc was formed just at the side of the shell where the observed HI density is higher. It was just assumption by Westerlund and Mathewson (1966) to explain the "arc of blue stars" in the LMC4 region now known as the Quadrant arc. However, this suggestion does not work just to explain the LMC4 arcs, for the new data demonstrated the Quadrant is deep inside the LMC4 HI hole. The rather similar complex in M83 was suggested to arise in the result of the HVC impact (Comeron, 2001), and this may be the case for the LMC arc complex as well (Efremov, 2002).

\section{Concluding remarks}

Putting the things together, we hypothesize that the hexagonal appearance might be the transient property of persistent vortices (of wide size range), which are suffered from the ram pressure. The straight segments of the rims might be a result of the the local flattening of the shock front on the space scale of local radius of curvature what might, in turn, be due to stability of a flat shock wave against any weak perturbations that disturb its front surfaces (Chernin, 1999). If the star formation occurred in the shock wave while its front was flat, the respective part of the rim of the stellar system would preserve the hexagonal shape. The flatenning of the shock wave fronts seen in galactic spiral arms (Chernin 1999) and now in other formations worth to be investigated. May be the Chandra data for clusters of galaxies will find something of the kind.

However, the origin of most round or arc-shaped complexes is still a problem. Considering that both stellar arcs in NGC 300 and M33 are the only such features in these galaxies and associated with the most unusual in the respective galaxy X-ray sources, both well isolated, the chance coincidence seems to be improbable. We believe that these X-ray objects, P42 and X-4 (especially P42), will be proved to be the remnants of Hypernovae. The estimated young age of the M33 X-4 SNR (Okada et al., 2001) 
is surely incompatible with our suggestion, yet the source should be studied in more details. Both these arcs are rather irregular, unlike other arcs discussed here.

The similar origin is possible also for the LMC4 arcs, yet there are no X-ray sources located at the suitable positions in respect to the arcs. The X-ray binaries concentrate mostly to North of the LMC4 supershell, near the NGC 1978 cluster. The origin of the LMC arcs in the result of the ram pressure to the surface of impacting clouds seems to be more probable, considering also the similar opening angles of these one-sided arcs. The main problem is that there are at least 2 and probably 5 arcs close to each other; it is a difficulty for any hypothesis, as well as the different orientations and ages. The Hodge object is enigmatic also, with a couple of concentric semiarcs, suggesting action of a central pressure yet having nor an evident source, neither an age - space pattern (see Fig. 1 here and Fig. 9 in Larsen et al., 2002). The vortex motions might confine the young stars within the round complex; however, the sharp arc of the Western rim suggests the one-sided ram pressure action. Note that the drift of the complex might explain the absence of the HI hole around it, what is very strange considering the complex contains the cluster most suitable to form a supershell. May be the understanding of these features will come from an unexpected side, such as the dark matter presence, or something else like this...

One may wonder how frequent are the round peculiar formations in galaxies. We were able to find a dozen only (Efremov 2001). The Hodge object in NGC 6946 was the only result of the special searches for the features similar to the giant arcs in the LMC4 region (P.Hodge, private communication). The appearance of this complex under different resolutions and contrasts suggests that many similar features may not be remarked. Under the low resolution, and in the distant galaxies they are practically star-like (the best examples are the round complexes in M51 and especially in NGC 1232, see Fig. 12), whereas under the low contrast (such as in the Sandage-Bedke atlas) the encompassing circular rim is unnoted. This is also the case for the stellar arcs even so large as in the LMC4 region. The rather similar in size complex in M83 (Comeron 2001) includes also two giant arcs (Fig. 12), which are seen only in the best resolution images (Efremov, 2001). 
At any rate, these peculiar entities are curious and elegant, and their interpretation may have the far reaching implications. Some were suggested in this paper.

\section{Acknowledgments}

I am very grateful to A.Chernin for the comments on the paper draft and many useful discussions, and to P.Korchagin for comments on the theoretical motions of a solitary vortex in a galaxy. The using of the NASA Astrophysics Data Systems, the LANL electronic preprint service, the Digital Sky Survey, the US Naval Observatory Flagstaff Station Integrated Image and Catalogue Archive Service, and also the HST and ESO Press Releases was necessary to carry out this work and is thankfully noted. Thanks are due also to S.Larsen and I.Karachentsev for the images obtained with the NOT and the BTA. The support from grants RFBR 00-02-17804 and 00-15-96627 is appreciated.

\section{REFERENCES}

Allison M., Godfrey D. A., Beebe, R. F. (1990) Science, 247, 1061,

Block D.L., Puerari I., Frogel J.A. et al. (1999) Astron, Sp. Sci., 269, 5.

Bonnarel F., Boulesteix J., Georgelin Y.P. et al. (1988), A\&A, 189, 59.

Bothun G.D. (1986) AJ, 91, 507.

Bureau M., Carignan C. (2002) AJ, 123, 131.

Buta R., 1990, ApJ, 356, 87

Buta R., Combes F., 1996, Fund. Cosm. Phys., 17, 95

Chernin A.D. (1996), Vistas in Astr. 40, 257.

Chernin A.D., 1999, MNRAS 308, 321

Chernin A.D., 2000, MNRAS 318, L7.

Chernin A.D., Zasov A.V., Archipova V.P., Kravtsova A. S., (2000) in: Galaxy disks and disk galaxies", eds. J.G.Funes and E.M.Corsini, ASP Conf. Ser., Vol. 230, p. 147.

Comeron F. (2001), A\&A, 365, 417.

Chu, Y.-H. (1999), Ap. Sp Sci., 269-270, 441. 
Dubrovskiy V. A. (2000) in: New Cosmological Data and the Values of the Fundamental Parameters, p. 52, IAU Symposium no. 201, Manchester.

Efremov Yu. N. (2001) Astron. Rep., 45, 769.

Efremov Yu. N. (2001b) astro-ph/0102161.

Efremov Yu. N. (2002) Astron. Rep., 46, \#10 (in press).

Efremov Yu. N., Pustilnik S.A., Kniazev A.Y. et al., (2002) A\&A, accepted = astro-ph/0205368.

Elmegreen B.G., Efremov Yu.N,, Larsen S.S. (2000) ApJ, 535, 748.

Elmegreen B.G., Kaufman M., Struck C. et al. (2000) AJ, 120, 630.

Gruendl R.A., Vogel S.N., Davis D.S., Mulchev J.S. (1993) ApJ, 413, L81

Hitchock J.C., Hodge P.W. (1968) ApJ, 152, 1067.

Hodge P.W. (1967) Publ. ASP, 79, 297.

Hummel E., Beck R. (1995) A\&A, 303, 691

Kamphuis J.J. (1993) Ph. D. Thesis, Univ. Groningen.

Kim S.C., Sung H., Lee M.G. (2002) astro-ph/0203032.

Korchagin V.I., Petviashvili V.I. (1985) Sov. Astr. Lett. 11, 121.

Korchagin V.I., Ryabtsev A.D. (1991) AA, 246, 368.

Kundu A. (2002) astro-ph/0206221.

Larsen S.S., Richtler T. (1999) A\&A, 345, 59.

Larsen S.S., Brodie J.P., Elmegreen B.G. et al. (2001) ApJ, 556, 801.

Larsen S.S., Efremov Yu.N., Elmegreen B.G et al. (2002) ApJ, 567, 896.

Lauer T.R., Gebhardt K., Richstone D. et al. (2002) astro-ph/0206122

McKee C.F. and Cowie L.L. (1975) ApJ, 195, 715.

Maxworthy T., Redekopp L.G. (1976) Icarus, 29, 261

Marel van den B.P., Alves D.R., Hardy E., Suntzev N.P. (2002), astro-ph/0205161

Meaburn J., Wang L., Bryce M. (1995) A\&A, 293, 532.

Merrifield M.R., Kuiken K. (1999) A\&A, 345, L47.

Noever D.A. (1994) A\&SS, 220, 65.

Okada Y., Takahashi H., Makishima K. (2001) PASJ, 53, 663.

Read A.M., Pietsch W., (2001) Astron. Astrophys., 373, 473. 
Redman M.P., Al-Mostafa Z.A., Meaburn J. et al. (1999) A\&A, 345, 943.

Ryder S.D., Purcell G., Davis D., Andersen V., (1997) Publ. AS Austr, 14, 81.

Sansom A.E., Reid I.N., Boisson C. (1988) MN RAS, 234, 247.

Santillan A., Franco J., Martos M., Kim J., (1999) ApJ 515, 657.

Shulman E., Bregman J.N. (1995) ApJ, 441, 568.

Wang Z. P., Wang L. Y. Liu, S. S. (2001) Acta Astr. Sinica, 42, 397.

Westerlund B.E., Mathewson D.S. (1966) MNRAS, 131, 371.

White R.E., Sarazin C.L., Kulkarni S.R. (2002), ApJ, 571, L23.

Whitmore B.C., Bell M. (1988) ApJ, 324, 741.

Yun M.S., Hibbard J.E. (2001) ApJ, 550, 104. 


\section{Figure captions}

Fig 1. The images of the Hodge object in NGC 6946. At the top - the segment of the HST WF camera image. Note the symmetric arcs of clusters "behind" (at the left) of the complex - the stellar relicts of von Karman vortex street? At the bottom - the edited NOT image, with the encompassing circle added. Note slightly different orientation; Nord is up in the NOT image.

Fig. 2. The map of HI holes in NGC 6946 (Kamphuis 1993) overlayed by the galaxy image map from USNO Image and Catalogue Archive Service. The Bubble complex is near \#13, looking quite similar to the bright foreground star. North is up.

Fig. 3. The ram pressure shaped NGC 7421 (top) and NGC 2276 (bottom) galaxies. At the left HI (NGC 7421) and $1.79 \mathrm{GHz}$ (NGC 2276) isolines, at the right - the DSS images. North is up. See the text.

Fig. 4. The DDO 165 galaxy images from the plate of 6-m telescope, courtesy of I.Karachentsev.

Fig. 5. The SNR N63 in the LMC (Chu, 1999). The isolines of X-ray emission are shown with the direction of the LMC proper motion added. North is up.

Fig. 6. The Jovian Red Spot in 1979. See the text.

Fig. 7. The samples of galaxies from the Hubble Deep Fields, probably shaped by the ram pressure. The top row $-120 \mathrm{~S}$ and $293 \mathrm{~N}$, the middle row $-222 \mathrm{~S}$ and $41 \mathrm{~N}$, the bottom row $-255 \mathrm{~N}$ and $96 \mathrm{~N}$. See the text.

Fig. 8. The left row - the Western (top) and Southern (Efremov, 2001) dark rings in M83 (the VLT ESO images); the NGC 4449 ring (bottom). The right row - the dark rings in NGC 6946 (top, NOT image); the dark ring in NGC 2207; the peculiar complex, which includes the elliptical dark ring, in NGC 2207 (bottom). The last two HST images are from Elmegreen et al. (2000).

Fig. 9. The hexagonal NGC 1637 galaxy (the edited DSS image).

Fig. 10. The Mices (NGC 4676AB) galaxies. It is the overlay of the HST ASC image and CO isolines from Yun and Hibbard (2001). North is up.

Fig. 11. At the top - the system of giant stellar arcs in the LMC4 region (the edited image based on the Boyden Observatory photograph obtained by H.Shapley; courtesy of 
P.Hodge and K.Olsen). At the bottom left- the AS102 arc in NGC 300 and the position of the X-ray source P42 (X-ray isolines) overlayed (the NOT image by S.Larsen). At the bottom right - the IC133 complex in M33 (DSS), with the position of M33 X-4 source shown as the yellow circle.

Fig. 12. The left row: the Bubble complex in NGC 6946 (top, HST PC); the round complex ( $300 \mathrm{pc}$ in size, around the bright cluster), 1.5' West of the center of M51 (middle, HST PC); the round complex (the very center of the image) inside the Northern spiral arm of NGC 1232 (bottom, VLT). The right row: the NE segment of the LMC, 30 Dor nebula is at the middle bottom (top); the LMC4 region; the SE complex in M83 with two arcs of clusters inside (VLT). 
This figure "fig1.jpg" is available in "jpg" format from: http://arxiv.org/ps/astro-ph/0206408v1 
This figure "fig2.jpg" is available in "jpg" format from: http://arxiv.org/ps/astro-ph/0206408v1 
This figure "fig3.jpg" is available in "jpg" format from: http://arxiv.org/ps/astro-ph/0206408v1 
This figure "fig4.jpg" is available in "jpg" format from: http://arxiv.org/ps/astro-ph/0206408v1 
This figure "fig5.jpg" is available in "jpg" format from: http://arxiv.org/ps/astro-ph/0206408v1 
This figure "fig6.jpg" is available in "jpg" format from: http://arxiv.org/ps/astro-ph/0206408v1 
This figure "fig7.jpg" is available in "jpg" format from: http://arxiv.org/ps/astro-ph/0206408v1 
This figure "Fig8.jpg" is available in "jpg" format from: http://arxiv.org/ps/astro-ph/0206408v1 
This figure "fig9.jpg" is available in "jpg" format from: http://arxiv.org/ps/astro-ph/0206408v1 
This figure "fig10.jpg" is available in "jpg" format from: http://arxiv.org/ps/astro-ph/0206408v1 
This figure "Fig11.jpg" is available in "jpg" format from: http://arxiv.org/ps/astro-ph/0206408v1 
This figure "fig12.jpg" is available in "jpg" format from: http://arxiv.org/ps/astro-ph/0206408v1 